Original Research Paper

\title{
Existence Results for a Class of Nonlinear Hadamard Fractional with $p$-Laplacian Operator Differential Equations
}

\author{
Samah Horrigue \\ Faculty of Science, University of Jeddah, Saudia Arabia
}

\author{
Article history \\ Received: 29-03-2021 \\ Revised: 01-05-2021 \\ Accepted: 10-05-2021 \\ E-mail: samah.horrigue @ fst.rnu.tn
}

\begin{abstract}
In this study, we are concerned with the existence and uniqueness of solution for some nonlinear Hadamard fractional differential equations. Our results are based on different classical fixed point theorems. Some useful examples are presented in order to illustrate the validity of our main results.
\end{abstract}

Keywords: Hadamard Fractional Derivative, Integral Equations, $p$ Laplacian Operator, Fixed Point Theorems

\section{Introduction}

Recently, fractional differential equations have been acquired much attention due to its applications in a number of fields such as physics, mechanics, chemistry, biology, signal and image processing, see for example the books (Baleanu et al., 2012; Kilbas et al., 2006; Lakshmikantham et al., 2009; Yang et al., 2015).

Some recent works on fractional differential equations involving Riemann Liouville and Caputo-type fractional derivatives are studied using nonlinear analysis methods such as Krasnoselskii fixed-point Theorems (Agarwal and O'Regan, 1998; Ghanmi and Horrigue, 2018; Guo et al., 2007; Guo et al., 2008), Leray-Schauder alternative (Ghanmi and Horrigue, 2019; Qi et al., 2017), sub-solution and super-solution methods (Wang et al., 2019; Mâagli et al., 2015) and iterative techniques (Liu et al., 2013).

Hadamard (1892) introduced an important fractional derivative, which differs from the above-mentioned ones because its definition involves logarithmic function of arbitrary exponent and named as Hadamard derivative. In the last few decades many authors are paying more and more attention to fractional differential equation involving Hadamard derivative, the study of the topic is still in its primary stage. For details and recent developments on Hadamard fractional differential equations, see (Huang and Liu, 2018; Wang et al., 2018; Zhai et al., 2018) and references therein. Recently, some researches have extensively interested in the study of the fractional differential equations with $p$-Laplacian operators see for examples (Chamekh et al., 2018; Ding et al., 2015).

From the above review of the literature concerning fractional differential equations, most of the authors investigated only the existence of solutions or positive solutions for Hadamard fractional differential equations without considering the $p i$-Laplacian operator. A very few authors established results along with $p$-Laplacian operator, us example in (Wang and Wang, 2016), the authors considered the following nonlinear Hadamard fractional differential problem:

$$
\left\{\begin{array}{l}
D^{\sigma}\left(\phi_{p}\left(D^{\alpha} u(t)\right)\right)=f(t, u(t)), \quad t \in(1, T), \\
u(T)=\lambda I^{\gamma} u(\eta), \quad D^{\alpha} u(1)=0, \quad u(1)=0,
\end{array}\right.
$$

where fore an appropriate $\xi, D^{\xi}$ is the Hadamard fractional derivative of order $\xi, 1<\alpha \leq 2,0<\sigma \leq 1, \gamma>0, \lambda \in \mathbb{R}$ and $f \in C([1, T] \times \mathbb{R}, \mathbb{R})$. By using the Schauder fixed point Theorem, the existence of solutions is obtained. In Li and Lin (2013), the authors used the Guo-Krasnosel'skii fixed point Theorem to prove the existence and uniqueness of positive solutions of the following Hadamard fractional boundary value with $p$-Laplacian operator:

$$
\left\{\begin{array}{l}
D^{\sigma}\left(\phi_{p}\left(D^{\alpha} u(t)\right)\right)=f(t, u(t)), \quad t \in(1, e), \\
u(1)=u^{\prime}(1)=u^{\prime}(e)=0, \quad D^{\alpha} u(1)=D^{\alpha} u(e)=0
\end{array}\right.
$$

where, $2<\alpha \leq 3,1<\sigma \leq 2, f \in C([1, e] \times[0, \infty),[0, \infty))$ and the function $\varphi_{p}(p>1)$, is called $p$-Laplacian and is defined in $\mathrm{R}$ by $\varphi_{p}(s)=|s|^{p-2} s$. The authors in Zhang et al. (2018) established some existence of positive solutions for the following nonlinear Hadamard fractional differential equations with $p$-Laplacian operator:

$$
\left\{\begin{array}{l}
D^{\sigma}\left(\phi_{p}\left(D^{\alpha} u(t)\right)\right)=f(t, u(t)), t \in(1, e), \\
u(1)=u^{\prime}(1)=u^{\prime}(e)=0, D^{\alpha} u(1)=0, \\
\phi_{p}\left(D^{\alpha} u(e)\right)=\mu \int_{1}^{e} \phi_{p}\left(D^{\alpha} u(t)\right) \frac{d t}{t},
\end{array}\right.
$$


where, $2<\alpha \leq 3,1<\sigma \leq 2,0 \leq \mu<\sigma$ and $\mathrm{f} \in \mathrm{C}([1, \mathrm{e}] \times \mathbb{R}, \mathbb{R})$.

Motivated by the above mentioned papers, in this study, we study the following fractional Hadamard problem:

$$
\left\{\begin{array}{l}
D^{\sigma}\left(\phi_{p}\left(D^{\alpha} x\right)\right)(t)=f(t, x), \\
x(1)=\phi_{p}\left(D^{\alpha} x\right)(1)=0, \\
A_{1} I^{\gamma_{1}} x\left(\eta_{1}\right)+B_{1} x(e)=c_{1}, \quad 0<\gamma_{1} \\
A_{2} I^{\gamma_{2}} x\left(\phi_{p}\left(D^{\alpha} x\right)\right)\left(\eta_{2}\right)+B_{2} \phi_{p}(e)=c_{2}, \quad 0<\gamma_{2}
\end{array}\right.
$$

where, $\alpha$ and $\sigma$ are in $(1,2], \eta_{1}$ and $\eta_{2}$ are in $(1, e), A_{1}, A_{2}$, $B_{1}, B_{2}, c_{1}$ and $c_{2}$ are fixed real numbers.

For the sake of computational convenience, we set:

$$
\begin{aligned}
& \Delta_{1}=B_{2}+\frac{A_{2} \Gamma(\sigma)}{\Gamma\left(\gamma_{2}+\sigma\right)}\left(\log \eta_{2}\right)^{\gamma_{2}+\sigma-1}, \\
& \Delta_{2}=B_{1}+\frac{A_{1} \Gamma(\sigma)}{\Gamma\left(\gamma_{1}+\alpha\right)}\left(\log \eta_{1}\right)^{\gamma_{1}+\alpha-1} .
\end{aligned}
$$

And we assume the following conditions.

$\left(H_{1}\right)$ There exist nonnegative functions $a(t), b(t) \in$ $C([1, e], \mathbb{R})$, such that:

$$
\begin{array}{rl}
|f(t, x)| \leq a & a(t)+b(t)|x|^{p-1}, \text { for each } t \in[1, e] \text { and } x \in \square . \\
& \left(H_{2}\right) \Theta_{1}+\Theta_{2}<1, \text { where } \\
\Theta_{1}=\left(\frac{\|b\| \infty}{\Gamma(\sigma+1)}\right)^{q-1} \frac{\left(2^{q-1}-1\right) \Gamma(\sigma(q-1)+1)}{\Gamma(\alpha+\sigma(q-1)+1)} \\
\left(1+\frac{\left|A_{1}\right|}{\left|\Delta_{2}\right|}+\left(2^{q-1}-1\right)\left(\frac{\left|A_{2}\right|+\left|B_{2}\right|}{\left|\Delta_{1}\right|}\right)\right)^{q-1}
\end{array}
$$

and:

$$
\Theta_{2}=\frac{\|b\| \infty}{\Gamma(\sigma+1) \Gamma(\alpha+1)\left|\Delta_{2}\right|}\left(\left|B_{1}\right|+\frac{\left(\left|A_{2}\right|+\left|B_{2}\right|\right)}{\left|\Delta_{1}\right|}\right) .
$$

$\left(H_{3}\right)$ There exist a positive continuous nondecreasing function $g$ on $[0, \infty)$ and a function $p \in C\left([1, e], \mathrm{R}^{+}\right)$such that:

$$
|f(t, x)| \leq p(t) g(\|x\|) \text { foreach }(t, u) \in[1, e] \times \square
$$

$\left(H_{4}\right)$ there exists $M_{2}>0$, such that:

$$
\frac{M_{2}}{\frac{w_{1}}{\Gamma^{q-1}(\sigma+1)}\|p\|_{\infty}^{q-1} g\left(M_{2}\right)^{q-1}+w_{2}\|p\|_{\infty} g\left(M_{2}\right)+w_{3}}>1,
$$

where $w_{1}, w_{2}$ and $w_{3}$ is given respectively by:

$$
\begin{aligned}
& w_{1}=\frac{\Gamma(\sigma(q-1)+1)}{\Gamma(\alpha+\sigma(q-1)+1)}\left[1+\frac{\left|A_{1}\right|}{\left|\Delta_{2}\right|}\right] \\
& +\frac{\left(2^{q-1}-1\right)\left(\left|A_{2}\right|+\left|B_{2}\right|\right)^{q-1}}{\left|\Delta_{1}\right|^{q-1}} \\
& {\left[1+\frac{\left|A_{1}\right| \Gamma(\sigma(q-1)+1)}{\left|\Delta_{2}\right| \Gamma(\alpha+\sigma)(q-1)+1}\right],}
\end{aligned}
$$

$$
w_{2}=\frac{\left|B_{1}\right| \Gamma(\sigma+1)}{\Gamma(\alpha+\sigma+1)}+\left(\frac{\left|A_{2}\right|+\left|B_{2}\right|}{\Gamma(\sigma+1) \Gamma(\alpha+1)\left|\Delta_{1}\right|}\right)
$$

and:

$$
\begin{aligned}
& w_{3}=\frac{\left|c_{1}\right|}{\left|\Delta_{2}\right|}+\frac{\left(2^{q-1}-1\right)^{2}\left|c_{2}\right|^{q-1}}{\left|\Delta_{1}\right|^{q-1}}\left(1+\frac{\left|A_{1}\right|}{\left|\Delta_{2}\right|}\right) \\
& +\frac{\left|c_{2} \| B_{1}\right|}{\left|\Delta_{1} \Delta_{2}\right| \Gamma(\alpha+1) \Gamma(\sigma+1)} .
\end{aligned}
$$

The main results of this study are summarized in the following theorems.

\section{Theorem 1.1}

Let $q \geq 2$. If $f \in C([1, e] \times \mathbb{R}, \mathbb{R})$ such that hypothesis $\left(H_{1}\right)$ and $\left(H_{2}\right)$ are satisfied, then the Hadamard fractional boundary value problem (1.1) has a unique solution.

\section{Theorem 1.2}

If $f \in C([1, e] \times \mathbb{R}, \mathbb{R})$ and if hypothesis $\left(H_{3}\right)$ and $\left(H_{4}\right)$ are fulfilled. Then the fractional boundary value problem (1.1) has at least one solution.

This study is organized as follows, in Section 2 we present some preliminaries and usefully results which will be used in the proofs of the main results. Section 3 is devoted to the proof of Theorem 1.1 and Theorem 1.2. In Section 4, we present some important examples in order to illustrate the main results of this article.

\section{Preliminaries}

In this section, we recall some results and we prove key lemmas which we will use later in section 3. Also, we give some definitions and properties related on Hadamard fractional calculus, we refer the reader to Kilbas et al. (2006) for more details.

\section{Definitions 2.1}

The Hadamard fractional integral of order $q>1$ for a function $g:[1, \infty) \rightarrow \mathbb{R}$ is defined as:

$$
I^{q} g(t)=\frac{1}{\Gamma(q)} \int_{1}^{t}\left(\log \frac{t}{s}\right)^{q-1} \frac{g(s)}{s} d s
$$

$$
=\frac{1}{\Gamma(q)}\left\{(\log (t))^{q} \int_{0}^{1}(1-s)^{q-1} g\left(e^{s \log (t)}\right) d s\right\},
$$

where, $\log ()=.\log _{e}($.$) :$

- The Hadamard derivative of fractional order $\alpha$ for a function $g:[1, \infty) \rightarrow \mathbb{R}$ is given by: 


$$
D^{\alpha} g(t)=\left(t \frac{d}{d t}\right)^{n} I^{n-\alpha}
$$

where, $n-1<\alpha<n, n=[\alpha]+1,[\alpha]$ denotes the integer part of the real number $\alpha$.

\section{Lemma 2.1}

[12] For any $t \in[1, e], c \in \mathbb{R}$ and any constants $\alpha, \sigma$ in $[1,2]$, we have:

$$
\begin{aligned}
& I^{\alpha}(c)(t)=c I^{\alpha} 1(t)=c \frac{1}{\Gamma(\alpha)} \\
& \int_{1}^{t}\left(\log \frac{t}{s}\right)^{\alpha-1} \frac{d s}{s}=\frac{c(\log t)^{\alpha}}{\Gamma(\alpha+1)} . \\
& I^{\alpha}\left[(\log (.))^{\sigma}\right](t)=\frac{1}{\Gamma(\alpha)} \\
& \int_{1}^{t}\left(\log \frac{t}{s}\right)^{\alpha-1} \frac{[\log (s)]^{\sigma} d s}{s}=\frac{(\log t)^{\alpha+\sigma} \Gamma(\sigma+1)}{\Gamma(\alpha+\sigma+1)} .
\end{aligned}
$$

We use also the following property:

If $q>2$ and $\max (|x|,|y|) \leq R$, then:

$$
\left|\Phi_{q}(x)-\Phi_{q}(y)\right| \leq(q-1) R^{q-2}|x-y| .
$$

The following elementary relation is usefully:

If $n>1$, then for all positive numbers $a$ and $b$, we have:

$$
(a+b)^{n} \leq\left(2^{n}-1\right)\left(a^{n}+b^{n}\right) .
$$

\section{Lemma 2.2}

Kilbas et al. (2006) Let $q>0$ and $x \in C[1, \infty) \cap L^{1}[1, \infty)$. Then the Hadamard fractional differential equation $D^{q} x(t)$ $=0$ has the solution:

$$
x(t)=\sum_{i=1}^{n} c_{i}(\log t)^{q-i},
$$

and the following formula holds:

$$
I^{q} D^{q} x(t)=x(t)+\sum_{i=1}^{n} c_{i}(\log t)^{q-i}
$$

where $c_{i} \in \mathbb{R}, i=1,2, \ldots, n$ and $n-1<q<n$.

\section{Theorem 2.3}

Given $y \in C([1, e], \mathbb{R})$, the unique solution of the problem:

$$
\left\{\begin{array}{l}
D^{\sigma}\left(\phi^{p}\left(D^{\alpha} x(t)\right)\right)=f(t, x), \\
x(1)=\phi^{p}\left(D^{\alpha} x(1)\right)=0, \\
A_{1} I^{\gamma_{1}} x\left(\eta_{1}\right)+B_{1} x(e)=c_{1}, \\
A_{2} I^{\gamma_{2}} x\left(\phi^{p}\left(D^{\alpha} x\right)\right)\left(\eta_{2}\right)+B_{2} \phi_{p}\left(D_{\alpha} x\right)(e)=c_{2},
\end{array}\right.
$$

is given by:

$$
x(t)=I^{\alpha} \phi_{q}\left[I^{\sigma} f(t, x)+c_{3}(\log (t))^{\sigma-1}\right]+c_{4}(\log t)^{\alpha-1},
$$

where, $c_{3}$ and $c_{4}$ are given by:

$c_{3}=\frac{c_{2}-A_{2} I^{\sigma+\gamma 2}\left(f\left(\eta_{2}, x\right)\right)-B_{2} I^{\sigma}(f(e, x))}{\Delta_{1}}$,

and:

$c_{4}=\frac{c_{1}-A_{1} I^{\alpha+\gamma 1} \phi_{q}\left[I^{\sigma}\left(f\left(\eta_{1}, x\right)\right)+c_{3}\left(\log \eta_{1}\right)^{\sigma-1}\right]\left(\eta_{1}\right)-B_{1} I^{\alpha}\left(I^{\alpha}(f(e, x))+c_{3}\right)}{\Delta_{2}}$,

where, $\Delta_{1}$ and $\Delta_{2}$ are given respectively in Eq. (1.2) and (1.3)

\section{Proof}

As argued in Kilbas et al. (2006), the Hadamard differential Equation in (2.8) can be written as:

$$
\phi_{p}\left(D^{\alpha} x(t)\right)=I^{\sigma}(f(t, x))+a(\log t)^{\sigma-1}+b(\log t)^{\sigma-2} .
$$

Since $\phi_{p}\left(D^{\alpha} x(1)\right)=0$, then $b=0$. So, we obtain:
$\phi_{p}\left(D^{\alpha} x(t)\right)=I^{\sigma} f(t, x)+a(\log t)^{\sigma-1}$.

By applying $I^{\gamma_{2}}$ on both sides of (2.12) for $t=\eta_{2}$ and using the property (2.5), we obtain:

$$
\begin{aligned}
& I^{\gamma_{2}}\left(\phi_{p}\left(D^{\alpha} x\left(\eta_{2}\right)\right)\right)=I^{\gamma_{2}+\sigma} f\left(\eta_{2}, x\right) \\
& +a \frac{\Gamma(\sigma)}{\Gamma\left(\eta_{2}+\sigma\right)}\left(\log \eta_{2}\right)^{\gamma_{2}+\sigma-1} .
\end{aligned}
$$


On the other hand, put $t=e$ in Equation (2.12), we obtain:

$\phi_{p}\left(D^{\alpha} x(e)\right)=I^{\sigma} f(e, x)+a$.

By combining Equations (2.13), (2.14) and the second boundary condition in (2.8), we obtain:

$$
\begin{aligned}
& \left.c_{2}=A_{2} I^{\gamma_{2}+\sigma} f\left(\eta_{2}, x\right)\right)+a\left[A_{2} \frac{\Gamma(\sigma)}{\Gamma\left(\gamma_{2}+\sigma\right)}\left(\log \eta_{2}\right)^{\eta_{2}+\sigma-1}+B_{2}\right] \\
& +B_{2} I^{\sigma} f(e, x) .
\end{aligned}
$$

Therefore:

$$
a=\frac{c_{2}-A_{2} I^{\gamma_{2}+\sigma} f\left(\eta_{2}, x\right)-B_{2} I^{\sigma} f(e, x)}{\Delta_{1}}=: c_{3}
$$

where:

$$
\Delta_{1}=B_{2}+\frac{A_{2} \Gamma(\sigma)}{\Gamma\left(\gamma_{2}+\sigma\right)}\left(\log \eta_{2}\right)^{\gamma 2+\sigma-1} .
$$

Then, the solution can be written us follows:

$x(t)=I^{\alpha}\left\{\varphi_{q}\left[I^{\sigma} f(t, x)+c_{3}(\log (t))^{\sigma-1}\right]\right\}+a^{0}(\log t)^{\alpha-1}+b^{0}(\log t)^{\alpha-2}$.

Since $x(1)=0$, then $b^{\prime}=0$ and we get:

$$
x(t)=I^{\alpha}\left\{\phi_{q}\left[I^{\sigma} f(t, x)+c_{3}(\log t)^{\sigma-1}\right]\right\}+a^{\prime}(\log t)^{\alpha-1} .
$$

Now, if we apply $I^{\gamma_{1}}$ to (2.16) and we replace $t$ by $\eta_{1}$, then, using the property (2.5), we obtain:

$$
\begin{aligned}
& I^{\gamma_{1}}\left(x\left(\eta_{1}\right)\right)=I^{\gamma_{1}+\alpha}\left[\phi_{q}\left\{I^{\sigma} f\left(\eta_{1}, x\right)+c_{3}\left(\log \eta_{1}\right)^{\sigma-1}\right\}\right] \\
& +a^{\prime} \frac{\Gamma(\alpha)}{\Gamma\left(\eta_{1}+\alpha\right)}\left(\log \eta_{1}\right)^{\gamma_{1}+\alpha-1} .
\end{aligned}
$$

On the other hand, Equation (2.16) with $t=e$, yields to:

$$
x(e)=I^{\alpha}\left\{\phi_{q}\left[I^{\sigma} f(e, x)+c_{3}\right]\right\}+a^{\prime}
$$

Finally, by combining Equations (2.17), (2.18) with the first boundary condition $c_{1}$, we obtain:

$$
\begin{aligned}
& c_{1}=A_{1} I^{\gamma_{1}+\alpha}\left[\phi_{q}\left(I^{\sigma} f\left(\eta_{1}, x\right)+c_{3}\left(\log \eta_{1}\right)^{\sigma-1}\right)\right] \\
& +a^{\prime}\left[A_{1} \frac{\Gamma(\alpha)}{\Gamma\left(\gamma_{1}+\alpha\right)}\left(\log \eta_{1}\right)^{\eta_{1}+\sigma-1}+B_{1}\right] \\
& +B_{1} I^{\alpha}\left[\phi_{q}\left(I^{\sigma} f(e, x)+c_{3}\right)\right] .
\end{aligned}
$$

It follows that:

$a^{\prime}=\frac{c_{1}-A_{1} I^{\gamma_{1}+\alpha}\left[\phi_{q}\left(I^{\sigma} f\left(\eta_{1}, x\right)+c_{3}\left(\log \eta_{1}\right)^{\sigma-1}\right)\right]-B_{1} I^{\alpha}\left[\phi_{q}\left(I^{\sigma} f(e, x)+c_{3}\right)\right]}{\Delta_{2}}=: c_{4}$

where:

$$
\Delta_{2}=B_{1}+\frac{A_{1} \Gamma(\alpha)}{\Gamma\left(\gamma_{1}+\alpha\right)}\left(\log \eta_{1}\right)^{\gamma_{1}+\alpha-1} .
$$

Substituting the values of $c_{3}$ and $c_{4}$ in (2.16), we obtain (2.9). This completes the proof.

To prove the main results of this study, we recall the following theorems.

\section{Theorem 2.4}

Smart (1974) Let $X$ be a Banach space. If the operator $T: X \rightarrow X$ is completely continuous and if the set:

$$
V=\{u \in X \mid u=\mu T u, 0<\mu<1\},
$$

is bounded. Then $T$ has a fixed point in $X$.

\section{Theorem 2.5}

Granas and Dugundji (2003) Let $X$ be a Banach space, $C$ be a closed, convex subset of $X, U$ be an open subset of $C$ and $0 \in C$. Suppose that the operator $F: U \rightarrow C$ is continuous and compact. Then either

(i). $F$ has a fixed point in $U$, or

(ii). There is $u \in \partial U$ and $\lambda \in(0,1)$, with $u=\lambda F(u)$.

\section{Proof of the Main Results}

This section is devoted to prove existence results for the nonlinear boundary value problem (1.1). Also, we shall prove existence and uniqueness results by using different methods. Let us de ne the operator $Q: C([1, e], \mathbb{R})$ $\rightarrow C([1, e], \mathbb{R})$ by:

$Q x(t)=I^{\alpha} \phi_{q}\left[I^{\sigma} f(t, x)+c_{3}(\log t)^{\sigma-1}\right]+c_{4}(\log t)^{\alpha-1}$, 
where $c_{3}$ and $c_{4}$ are given respectively by Equation (2.10) and (2.11). Notice that the existence of fixed points of the operator $Q$ is equivalent to the existence of solutions for problem (1.1).

\section{Proof of Theorem 1.1}

In this subsection, by using the well known Banach's fixed point Theorem, we present the existence and uniqueness result for Problem (1.1).

Let $B_{R}=\left\{x \in C([1, e], \mathbb{R}):\|x\|_{\infty} \leq R\right\}$. The proof is divided into tow steps.

Step 1: In this step, we will prove that the operator $Q$ is completely continuous. Let $O$ be an open bounded subset of $C([1, e], \mathbb{R})$. Since $f$ is continuous, then, the operator $Q$ is contunuous and so, $Q(O)$ is bounded. Next, we will show that $Q$ is equicontinuous.

Let $1 \leq t_{1}<t_{2} \leq e$, then, from Inequality (2.6) we obtain:

$$
\begin{aligned}
& \left|Q(x)\left(t_{2}\right)-Q(x)\left(t_{1}\right)\right| \\
& \leq \mid I^{\alpha}\left[\Phi_{q}\left\{I^{\sigma} f\left(x, t_{2}\right)+c_{3}\left(\log t_{2}\right)^{\sigma-1}\right\}-\Phi_{q}\right. \\
& \left.\left\{I^{\sigma} f\left(x, t_{1}\right)+c_{3}\left(\log t_{1}\right)^{\sigma-1}\right\}\left(t_{1}\right)\right] \mid \\
& +\mid c_{4}\left[\left(\log t_{2}\right)^{\alpha-1}-\left(\log t_{1}\right)^{\alpha-1}\right] \\
& \leq(q-1) R^{q-2}\left[I^{\alpha} I^{\alpha}(f)\left(x, t_{2}\right)-I^{\alpha} I^{\alpha}(f)\left(x, t_{1}\right)\right] \\
& +(q-1) R^{q-2}\left|c_{3}\right|\left[\left(\log t_{2}\right)^{\sigma-1}-\left(\log t_{1}\right)^{\sigma-1}\right] \\
& +\left|c_{4}\right|\left[\left(\log t_{2}\right)^{\alpha-1}-\left(\log t_{1}\right)^{\alpha-1}\right] .
\end{aligned}
$$

So, by using (2.1), (2.4) and the fact that $f$ is bounded, we get:

$$
\begin{aligned}
& \left|I^{\sigma}(f)(x, t)\right| \leq \frac{1}{\Gamma(\sigma)} \int_{1}^{t}\left(\log \frac{t}{s}\right)^{\sigma-1}\left|\frac{f(s, x(s))}{s}\right| d s \\
& \leq \frac{1}{\Gamma(\sigma)} \int_{1}^{t}\left(\log \frac{t}{s}\right)^{\sigma-1} \frac{M}{s} d s \leq \frac{M}{\Gamma(\sigma+1)}(\log t)^{\sigma},
\end{aligned}
$$

for some $M>0$. Hence, (2.5), implies that:

$$
\left|I^{\alpha} I^{\sigma} f(x, t)\right| \leq \frac{M(\log t)^{\alpha+\sigma}}{\Gamma(\alpha+\sigma+1)} .
$$

By combining (3.2) and (3.4), we obtain:

$$
\begin{aligned}
& \left|Q(x)\left(t_{2}\right)-Q(x)\left(t_{1}\right)\right| \\
& \leq \frac{(q-1) R^{q-2} M}{\Gamma(\alpha+\sigma+1)}\left[\left(\log t_{2}\right)^{\alpha+\sigma}-\left(\log t_{1}\right)^{\alpha+\sigma}\right]
\end{aligned}
$$

On the other hand, from (2.10) and (3.3), we obtain:

$$
\begin{aligned}
& \left|c_{3}\right| \leq \frac{\left|c_{2}\right|+\left|A_{2} I^{\sigma+\gamma_{2}} f\left(x, \eta_{2}\right)\right|+\left|B_{2} I^{\sigma} f(x, e)\right|}{\left|\Delta_{1}\right|} \\
& \leq \frac{\left|c_{2}\right|+\left|A_{2}\right| \frac{M}{\Gamma\left(\sigma+\gamma_{2}+1\right)}\left(\log \eta_{2}\right)^{\sigma+\gamma_{2}}+\left|B_{2}\right| \frac{M}{\Gamma(\sigma+1)}}{\left|\Delta_{1}\right|} \\
& \leq \frac{\left|c_{2}\right|+M \frac{\left|A_{2}\right|}{\Gamma\left(\sigma+\gamma_{2}+1\right)}\left(\log \eta_{2}\right)^{\sigma+\gamma_{2}}+\frac{\left|B_{2}\right|}{\Gamma(\sigma+1)}}{\left|\Delta_{1}\right|}
\end{aligned}
$$

Therefore using (2.6), (2.11) and (3.4), we get:

$$
\begin{aligned}
& \left|c_{4}\right| \leq \frac{\left|c_{1}\right|+\left|A_{1}\right|\left|(q-1) R^{q-2} I^{\alpha+\gamma_{1}}\left[I^{\sigma} f\left(\eta_{1}, x\right)+c_{3}\left(\log \eta_{1}\right)^{\sigma-1}\right]\right|+\left|B_{1}\right|\left|I^{\alpha}\left(I^{\sigma} f(e, x)+c_{3}\right)\right|}{\left|\Delta_{2}\right|} \\
& \leq \frac{\left|c_{1}\right|+\left|A_{1}\right|\left|(q-1) R^{q-2}\left[\frac{\left(\log \eta_{1}\right)^{\alpha+\sigma+\gamma_{1}} M}{\Gamma\left(\alpha+\sigma+\gamma_{1}+1\right)}+\frac{\left|c_{3}\right|\left(\log \eta_{1}\right)^{\alpha+\gamma_{1}+\sigma-1} \Gamma(\sigma-2)}{\Gamma\left(\alpha+\gamma_{1}+\sigma\right)}\right]\right|}{\left|\Delta_{2}\right|}+\frac{\left|B_{1}\right|\left[\frac{M}{\Gamma(\alpha+\sigma+1)}+\frac{c_{3}}{\Gamma(\alpha+1)}\right]}{\left|\Delta_{2}\right|} \\
& \leq \frac{\left|c_{1}\right|+M\left[(q-1) R^{q-2}\left|A_{1}\right|\left[\frac{\left(\log \eta_{1}\right)^{\alpha+\sigma+\gamma_{1}}}{\Gamma\left(\alpha+\sigma+\gamma_{1}+1\right)}\right]+\frac{\left|B_{1}\right|}{\Gamma(\alpha+\sigma+1)}\right]}{\left|\Delta_{2}\right|} \\
& +\frac{\left(\left|c_{2}\right|+M\left(\frac{A_{2}}{\Gamma\left(\sigma+\gamma_{2}+1\right)}\left(\log \eta_{2}\right)^{\sigma+\gamma_{2}}+\frac{\left|B_{2}\right|}{\Gamma(\sigma+1)}\right)\right)\left(\frac{\left(\log \eta_{1}\right)^{\alpha+\gamma_{1}+\sigma-1} \Gamma(\sigma-2)}{\Gamma\left(\alpha+\gamma_{1}+\sigma\right)}+\frac{1}{\Gamma(\alpha+1)}\right)}{\left|\Delta_{1} \Delta_{2}\right|} .
\end{aligned}
$$


Samah Horrigue / Journal of Mathematics and Statistics 2021, Volume 17: 61.72

DOI: 10.3844/ajabssp.2021.61.72

By combining (3.5), (3.6) and (3.7), one has:

$$
\lim _{t_{2} \rightarrow t_{1}}\left|Q(x)\left(t_{2}\right)-Q(x)\left(t_{1}\right)\right|=0 .
$$

Finally, the Arzelá-Ascoli Theorem implies that $Q$ : $C([1, e], \mathbb{R}) \rightarrow C([1, e], \mathbb{R})$ is completely continuous.

Step 2: In this step, we will pore that the set $D=\{x \in$ $C([1, e], \mathbb{R}): x=\mu Q(x), \mu \in(0,1)\}$ is bounded. From hypothesis $\left(H_{1}\right)$ and using $(2.7)$, we have:

$$
\begin{aligned}
& |x(t)|=|\mu(Q x)(t)| \\
& \leq \max _{t \in[1, e]}\left|I^{\alpha} \Phi_{q}\left(I^{\sigma}(f)(x, t)+c_{3}(\log t)^{\sigma-1}\right)+c_{4}(\log t)^{\alpha-1}\right| \\
& \leq \max _{t \in[1, e]}\left|I^{\alpha} \Phi_{q}\left(I^{\sigma}(A)(x, t)+c_{3}(\log t)^{\sigma-1}\right)+\right| c_{4}\left|(\log t)^{\alpha-1}\right| \\
& \leq \max _{t \in[1, e]}\left[I^{\alpha} \Phi_{q}\left(A\left(\frac{(\log t)^{\sigma}}{\Gamma(\sigma+1)}\right)+\left|c_{3}\right|(\log t)^{\sigma-1}\right)+\left|c_{4}\right|(\log t)^{\alpha-1}\right] \\
& \leq \max _{t \in[1, e]}\left[I^{\alpha}\left\{\left(\frac{A}{\Gamma(\sigma+1)}+\left|c_{3}\right|\right)^{q-1}(\log t)^{\sigma(q-1)}\right\}+\left|c_{4}\right|(\log t)^{\alpha-1}\right] \\
& \leq \max _{t \in[1, e]}\left[\left(\frac{A}{\Gamma(\sigma+1)}+\left|c_{3}\right|\right)^{q-1} \frac{(\log t)^{\alpha+\sigma(q-1)} \Gamma(\sigma(q-1)+1)}{\Gamma(\alpha+\sigma(q-1)+1)}+\left|c_{4}\right|(\log t)^{\alpha-1}\right] \\
& \leq\left(\frac{A}{\Gamma(\sigma+1)}+\left|c_{3}\right|\right)^{q-1} \frac{\Gamma(\sigma(q-1)+1)}{\Gamma(\alpha+\sigma(q-1)+1)}+\left|c_{4}\right| \\
& \leq\left(2^{q-1}-1\right)\left[\left(\frac{A}{\Gamma(\sigma+1)}\right)^{q-1}+\left|c_{3}\right|^{q-1}\right] \frac{\Gamma(\sigma(q-1)+1)}{\Gamma(\alpha+\sigma(q-1)+1)}+\left|c_{4}\right|,
\end{aligned}
$$

where, $A=\|a\|_{\infty}+\|\mathrm{b}\|_{\infty}\|x\|_{\infty}{ }^{p-1}$.
On the other hand, we have:

$\left|c_{3}\right| \leq \frac{\left|c_{2}\right|+\left|A_{2} I^{\sigma+\gamma_{2}} f\left(x, \eta_{2}\right)\right|+\left|B_{2} I^{\sigma} f(x, e)\right|}{\left|\Delta_{1}\right|}$

$\leq \frac{\left|c_{2}\right|+\left|A_{2}\right| \frac{A}{\Gamma\left(\sigma+\gamma_{2}+1\right)}\left(\log \eta_{2}\right)^{\sigma+\gamma_{2}}+\left|B_{2}\right| \frac{A}{\Gamma(\sigma+1)}}{\left|\Delta_{1}\right|}$

$\leq \frac{\left|c_{2}\right|+A\left(\frac{\left|A_{2}\right|}{\Gamma\left(\sigma+\gamma_{2}+1\right)}\left(\log \eta_{2}\right)^{\sigma+\gamma_{2}}+\frac{\left|B_{2}\right|}{\Gamma(\sigma+1)}\right)}{\left|\Delta_{1}\right|}$

$\leq \frac{\left|c_{2}\right|+A\left(\frac{\left|A_{2}\right|}{\Gamma\left(\sigma+\gamma_{2}+1\right)}+\frac{\left|B_{2}\right|}{\Gamma(\sigma+1)}\right)}{\left|\Delta_{1}\right|}$,

$\leq \frac{\left|c_{2}\right|}{\left|\Delta_{1}\right|}+\frac{A}{\left|\Delta_{1}\right| \Gamma(\sigma+1)}\left(\left|A_{2}\right|+\left|B_{2}\right|\right)$.

Then, using Inequality (2.7), we obtain

$\left|c_{3}\right|^{q-1} \leq\left(2^{q-1}-1\right)\left[\left(\frac{\left|c_{2}\right|}{\left|\Delta_{1}\right|}\right)^{q-1}+\left(\frac{A\left(\left|A_{2}\right|+\left|B_{2}\right|\right)}{\Gamma(\sigma+1)\left|\Delta_{1}\right|}\right)^{q-1}\right]$,

and: 
Therefore, it follows from (2.7), (3.8), (3.9) and (3.10), that:

$$
\begin{aligned}
& |x(t)|=|\mu(Q x)(t)| \\
& \leq\left(2^{q-1}-1\right)\left[\left(\frac{A}{\Gamma(\sigma+1)}\right)^{q-1}+\left(2^{q-1}-1\right)\left[\left(\frac{\left|c_{2}\right|}{\left|\Delta_{1}\right|}\right)^{q-1}+\left(\frac{A\left(\left|A_{2}\right|+\left|B_{2}\right|\right)}{\Gamma(\sigma+1)\left|\Delta_{1}\right|}\right)^{q-1}\right]\right] \frac{\Gamma(\sigma(q-1)+1)}{\Gamma(\alpha+\sigma(q-1)+1)} \\
& +\frac{\left|c_{1}\right|+\left(2^{q-1}-1\right)\left|A_{1}\right|\left[\left(\frac{A}{\Gamma(\sigma+1)}\right)^{q-1}+\left(\left|c_{3}\right|\right)^{q-1}\right] \frac{\Gamma(\sigma(q-1)+1)}{\Gamma\left(\alpha+\gamma_{1}+\sigma(q-1)+1\right)}}{\left|\Delta_{2}\right|} \\
& \left.+\frac{\left(\left|B_{1}\right| A\right)}{\Gamma(\sigma+1) \Gamma(\alpha+1)}+\frac{\left|c_{2}\right|}{\left|\Delta_{1}\right| \Gamma(\alpha+1)}+\frac{A}{\left|\Delta_{1}\right| \Gamma(\sigma+1) \Gamma(\alpha+1)}\left(\left|A_{2}\right|+\left|B_{2}\right|\right)\right) \\
& \leq\left(\frac{A}{\Gamma(\sigma+1)}\right)^{q-1} \frac{\left(2^{q-1}-1\right) \Gamma(\sigma(q-1)+1)}{\Gamma(\alpha+\sigma(q-1)+1)}\left(1+\frac{\left|A_{1}\right|}{\left|\Delta_{2}\right|}+\left(2^{q-1}-1\right)\left(\frac{\left(\left|A_{2}\right|+\left|B_{2}\right|\right)}{\left|\Delta_{1}\right|}\right)\right)^{q-1} \\
& +\frac{\left|c_{1}\right|}{\left|\Delta_{2}\right|}+\frac{\left|c_{2}\right|}{\Gamma(\alpha+1)\left|\Delta_{1} \Delta_{2}\right|}+\left(2^{q-1}-1\right)^{2}\left(\frac{\left|c_{2}\right|}{\left|\Delta_{1}\right|}\right)^{q-1}\left(1+\frac{\left|A_{1}\right|}{\left|\Delta_{2}\right|}\right) \frac{\Gamma(\sigma(q-1)+1)}{\Gamma(\alpha+\sigma(q-1)+1)} \\
& +\frac{A}{\Gamma(\sigma+1) \Gamma(\alpha+1)\left|\Delta_{2}\right|}\left(\left|B_{1}\right|+\frac{\left|A_{2}+\right|+\left|B_{2}\right|}{\left|\Delta_{1}\right|}\right) .
\end{aligned}
$$

Using the inequality (2.7), we have:

$$
\begin{aligned}
& A^{q-1}=\left(\|a\|_{\infty}+\|b\|_{\infty}\|x\|_{\infty}^{p-1}\right)^{q-1} \\
& \leq\left(\|a\|_{\infty}^{q-1}+\|b\|_{\infty}^{q-1}\|x\|_{\infty}\right) .
\end{aligned}
$$

Since $q \geq 2$, then, $p \leq 2$ and $\|x\|_{\infty}^{p-1} \leq\|x\|_{\infty}$. Thus, we have:

$$
\|x\|_{\infty}<\left(\Theta_{1}+\Theta_{2}\right)\|x\|_{\infty}+\Theta_{3},
$$

where:

$$
\begin{aligned}
& \Theta_{1}=\left(\frac{\|b\|_{\infty}}{\Gamma(\sigma+1)}\right)^{q-1} \frac{\left(2^{q-1}-1\right) \Gamma(\sigma(q-1)+1)}{\Gamma(\alpha+\sigma(q-1)+1)} \\
& \left(1+\frac{\left|A_{1}\right|}{\left|\Delta_{2}\right|}+\left(2^{q-1}-1\right)^{2}\left(\frac{\left|A_{2}\right|+\left|B_{2}\right|}{\left|\Delta_{1}\right|}\right)^{q-1}\right), \\
& \Theta_{2}=\frac{\|b\| \infty}{\Gamma(\sigma+1) \Gamma(\alpha+1)\left|\Delta_{2}\right|}\left(\left|B_{1}\right|+\frac{\left(\left|A_{2}\right|+\left|B_{2}\right|\right)}{\left|\Delta_{1}\right|}\right),
\end{aligned}
$$

and:

$$
\begin{aligned}
& \Theta_{3}=\frac{\left|c_{1}\right|}{\left|\Delta_{2}\right|}+\frac{\left|c_{2}\right|}{\Gamma(\alpha+1)\left|\Delta_{1} \Delta_{2}\right|}+\frac{\left(2^{q-1}-1\right) \Gamma(\sigma(q-1)+1)}{\Gamma(\alpha+\sigma(q-1)+1)} \\
& \left(\frac{\left|c_{2}\right|}{\left|\Delta_{1}\right|}\right)^{q-1}\left(\left(2^{q-1}-1\right)+\frac{\left|A_{1}\right|}{\left|\Delta_{2}\right|}\right)+\left(\frac{\|a\| \infty}{\Gamma(\sigma+1)}\right)^{q-1} \frac{\left(2^{q-1}-1\right) \Gamma(\sigma(q-1)+)}{\Gamma(\alpha+\sigma(q-1)+1)} \\
& \left(1 \frac{\left|A_{1}\right|}{\left|\Delta_{2}\right|}+\left(2^{q-1}-1\right)\left(\frac{\left|A_{2}\right|+\left|B_{2}\right|}{\left|\Delta_{1}\right|}\right)^{q-1}\right)+\frac{\|a\| \infty}{\Gamma(\sigma+1) \Gamma(\alpha+1)}\left(\left|B_{1}\right|+\frac{\left|A_{2}\right|+\left|B_{2}\right|}{\left|\Delta_{1}\right|}\right) .
\end{aligned}
$$

This together with condition $\left(H_{2}\right)$, gives $\|u\|_{\infty}<M_{1}$. That is $D$ is bounded, so the operator $Q$ has at lest one fixed point. Which implies that the problem (1.1) has at least one solution.

\section{Proof of Theorem 1.2}

In this subsection, by using Leray-Schauder's nonlinear alternative Theorem, we give the proof of Theorem 1.2. The proof is divided into several steps. Step 1: In this step, We prove that $Q$ maps bounded sets into equicontinuous sets of $C([1, e], \mathbb{R})$. Let $t_{1}, t_{2} \in[1, e]$ with $t_{1}<t_{2}$ and $x \in B_{r}$. Then, we have:

$$
\begin{aligned}
& I_{\sigma} f\left(x, t_{1}\right)-I_{\sigma} f\left(x, t_{2}\right) \\
& \leq \frac{g(r)\|p\|_{\infty}}{\Gamma(\sigma)}\left[\int_{1}^{t_{1}}\left(\log \frac{t_{1}}{s}\right)^{\sigma-1} \frac{d s}{s}-\int_{1}^{t_{2}}\left(\log \frac{t_{2}}{s}\right)^{\sigma-1} \frac{d s}{s}\right] \\
& \left.\leq \frac{g(r)\|p\|_{\infty}}{\Gamma(\sigma)}\left[\int_{1}^{t_{1}}\left(\log \frac{t_{1}}{s}\right)^{\sigma-1}-\left(\log \frac{t_{2}}{s}\right)^{\sigma-1}\right] \frac{d s}{s}-\int_{1}^{t_{2}}\left(\log \frac{t_{2}}{s}\right)^{\sigma-1} \frac{d s}{s}\right] \text {. }
\end{aligned}
$$


So, using Inequality (2.6), we obtain:

$\left|(Q x)\left(t_{1}\right)-(Q x)\left(t_{2}\right)\right|$

$\leq I_{\alpha} \Phi_{q}\left[I_{\sigma} f\left(x, t_{1}\right)+c_{3}\left(\log t_{1}\right)^{\sigma-1}\right]-I_{\alpha} \Phi_{q}\left[I_{\sigma} f\left(x, t_{2}\right)+c_{3}\left(\log t_{1}\right)^{\sigma-1}\right]+c_{4}\left[\left(\log t_{2}\right)^{\alpha-1}-\left(\log t_{2}\right)^{\alpha-1}\right]$

$\leq I_{\alpha}\left\{\Phi_{q}\left[I_{\sigma} f\left(x, t_{1}\right)+c_{3}\left(\log t_{1}\right)^{\sigma-1}\right]-\Phi_{q}\left[I_{\sigma} f\left(x, t_{2}\right)+c_{3}\left(\log t_{2}\right)^{\sigma-1}\right]\right\}+c_{4}\left[\left(\log t_{1}\right)^{\alpha-1}-\left(\log t_{2}\right)^{\alpha-1}\right]$

$\leq I_{\alpha}\left\{(q-1) r^{q-2}\left[I_{\sigma} f\left(x, t_{1}\right)-I_{\sigma} f\left(x, t_{2}\right)\right]+c_{3}\left[\left(\log t_{1}\right)^{\sigma-1}-\left(\log t_{2}\right)^{\sigma-1}\right]\right\}+c_{4}\left[\left(\log t_{1}\right)^{\alpha-1}-\left(\log t_{2}\right)^{\alpha-1}\right]$

$\left.\leq I_{\alpha}\left\{\frac{(q-1) r^{q-2} g(r\|p\|)}{\Gamma(\sigma)}\left[\int_{1}^{t_{1}}\left(\log \frac{t_{1}}{s}\right)^{\sigma-1}-\left(\log \frac{t_{2}}{s}\right)^{\sigma-1}\right] \frac{d s}{s}-\int_{t_{1}}^{t_{2}}\left(\log \frac{t_{2}}{s}\right)^{\sigma-1} \frac{d s}{s}\right]+c_{3}\left[\left(\log t_{1}\right)^{\sigma-1}-\left(\log t_{2}\right)^{\sigma-1}\right]\right\}+c_{4}\left[\left(\log t_{1}\right)^{\alpha-1}-\left(\log t_{2}\right)^{\alpha-1}\right]$

$\left.\leq I_{\alpha}\left\{\frac{(q-1) r^{q-2} g(r\|p\|)}{\Gamma(\sigma+1)}\left[\left(\log t_{1}\right)^{\sigma-1}-\left(\log t_{2}\right)^{\sigma-1}\right]-\left(\log \frac{t_{2}}{t_{1}}\right)^{\sigma}\right]+c_{3}\left[\left(\log t_{1}\right)^{\sigma-1}-\left(\log t_{2}\right)^{\sigma-1}\right]\right\}+c_{4}\left[\left(\log t_{1}\right)^{\alpha-1}-\left(\log t_{2}\right)^{\alpha-1}\right]$

$\left.\leq\left\{\frac{(q-1) r^{q-2} g(r\|p\|)}{\Gamma(\sigma+\sigma+1)}\left[\left(\log t_{1}\right)^{\alpha+\sigma-1}-\left(\log t_{2}\right)^{\alpha+\sigma-1}\right]-\left(\log \frac{t_{2}}{t_{1}}\right)^{\alpha+\sigma}\right]+c_{3}\left[\left(\log t_{1}\right)^{\sigma-1}-\left(\log t_{2}\right)^{\sigma-1}\right]\right\}+c_{4}\left[\left(\log t_{1}\right)^{\alpha-1}-\left(\log t_{2}\right)^{\alpha-1}\right]$.

Obviously the right-hand side of the above inequality tends to zero independently of $x \in B_{r}$ as $t_{1}-t_{2} \rightarrow 0$.

Step 2: In this step, we will prove that $Q$ maps bounded sets (balls) into bounded sets in $C([1, e], \mathbb{R})$.
Let $r>0, t \in[1, e]$ and $x \in B_{r}$, then, by using the hypothesis $\left(H_{3}\right)$ and the inequality (2.7), we obtain:

$$
\begin{aligned}
& |Q(x)(t)| \leq \max _{t \in[1, e]}\left|I^{\alpha} \phi_{q}\left[I^{\sigma} f(t, x)+c_{3}(\log t)^{\sigma-1}\right]+c_{4}(\log t)^{\alpha-1}\right| \\
& \leq \max _{t \in[1, e]}\left|I^{\alpha} \phi_{q}\left[\frac{1}{\Gamma(\sigma)} \int_{1}^{t}\left(\log \frac{t}{s}\right)^{\sigma-1} \frac{f(s, x)}{s} d s+c_{3}(\log t)^{\sigma-1}\right]+c_{4}(\log t)^{\alpha-1}\right| \\
& \leq \max _{t \in[1, e]}\left|I^{\alpha} \phi_{q}\left[\frac{\|p\|_{\infty} g\left(\|x\|_{\infty}\right)}{\Gamma(\sigma)} \int_{1}^{t}\left(\log \frac{t}{s}\right)^{\sigma-1} \frac{1}{s} d s+c_{3}(\log t)^{\sigma-1}\right]+c_{4}(\log t)^{\alpha-1}\right| \\
& \leq \max _{t \in[1, e]}\left|I^{\alpha} \phi_{q}\left[\frac{\|p\|_{\infty} g\left(\|x\|_{\infty}\right)(\log t)^{\sigma}}{\Gamma(\sigma+1)}+c_{3}(\log t)^{\sigma-1}\right]+c_{4}(\log t)^{\alpha-1}\right| \\
& \leq \max _{t \in[1, e]}\left|I^{\alpha}\left[\frac{\|p\|_{\infty} g\left(\|x\|_{\infty}\right)(\log t)^{\sigma}}{\Gamma(\sigma+1)}+c_{3}(\log t)^{\sigma-1}\right]^{q-1}+c_{4}(\log t)^{\alpha-1}\right| \\
& \leq \max _{t \in[1, e]}\left|I^{\alpha}\left[\frac{\|p\|_{\infty}^{q-1} g\left(\|x\|_{\infty}\right)^{q-1}(\log t)^{\sigma(q-1)}}{\Gamma(\sigma+1)^{q-1}}+c_{3}(\log t)^{(\sigma-1)(q-1)}\right]\left(2^{q-1}-1\right)+c_{4}(\log t)^{\alpha-1}\right| \\
& \leq \max _{t \in[1, e]} \|\left[\frac{\|p\|_{\infty}^{q-1} g\left(\|x\|_{\infty}\right)^{q-1}(\log t)^{\alpha+\sigma(q-1)} \Gamma(\sigma(q-1)+1)}{\Gamma(\alpha+\sigma(q-1)+1) \Gamma(\sigma+1)^{q-1}}+c_{3}(\log t)^{(\sigma-1)(q-1)}\right]\left(2^{q-1}-1\right)+c_{4} \mid \\
& \leq\left(2^{q-1}-1\right)\left[\frac{\|p\|_{\infty}^{q-1} g\left(\|x\|_{\infty}\right)^{q-1} \Gamma(\sigma(q-1)+1)}{\Gamma(\alpha+\sigma(q-1)+1) \Gamma(\sigma+1)^{q-1}}+c_{3}^{q-1}\right]+c_{4} .
\end{aligned}
$$


Samah Horrigue / Journal of Mathematics and Statistics 2021, Volume 17: 61.72

DOI: 10.3844/ajabssp.2021.61.72

In the other hand, we have:

$$
\begin{aligned}
& \left|c_{3}\right| \leq \frac{\left|c_{2}\right|+\left|A_{2} I^{\sigma+\gamma_{2}} f\left(x, \eta_{2}\right)\right|+\left|B_{2} I^{\sigma} f(x, e)\right|}{\left|\Delta_{1}\right|} \leq \frac{\left|c_{2}\right|+\left|A_{2}\right| \frac{\|p\|_{\infty} g\left(\|x\|_{\infty}\right)}{\Gamma\left(\sigma+\gamma_{2}+1\right)}\left(\log \eta_{2}\right)^{\sigma+\gamma_{2}}+\left|B_{2}\right| \frac{\|p\|_{\infty} g(\|x\| \infty)}{\Gamma(\sigma+1)}}{\left|\Delta_{1}\right|} \\
& \leq \frac{\left|c_{2}\right|+\|p\|_{\infty} g\left(\|x\|_{\infty}\right)\left(\frac{\left|A_{2}\right|}{\Gamma\left(\sigma+\gamma_{2}+1\right)}\left(\log \eta_{2}\right)^{\sigma+\gamma_{2}}+\frac{\left|B_{2}\right|}{\Gamma(\sigma+1)}\right)}{\left|\Delta_{1}\right|} \leq \frac{\left|c_{2}\right|+\|p\|_{\infty} g\left(\|x\|_{\infty}\right)\left(\frac{\left|A_{2}\right|}{\Gamma\left(\sigma+\gamma_{2}+1\right)}+\frac{\left|B_{2}\right|}{\Gamma(\sigma+1)}\right)}{\left|\Delta_{1}\right|} \\
& \leq \frac{\left|c_{2}\right|+\|p\|_{\infty} g\left(\|x\|_{\infty}\right)\left(\left|A_{2}\right|+\left|B_{2}\right|\right)}{\Gamma(\sigma+1)\left|\Delta_{1}\right|}, \\
& \left|c_{3}\right|^{q-1} \leq\left(2^{q-1}-1\right) \frac{\left|c_{2}\right|^{q-1}+\|p\|_{\infty}^{q-1} g\left(\|x\|_{\infty}\right)^{q-1}\left(\left|A_{2}\right|+\left|B_{2}\right|\right)^{q-1}}{\left(\Gamma(\sigma+1)\left|\Delta_{1}\right|\right)^{q-1}},
\end{aligned}
$$

and:

$$
\begin{aligned}
& \left|c_{4}\right|=\left|\frac{c_{1}-A_{1} I^{\alpha+\gamma_{1}} \phi_{q}\left[I^{\sigma} F\left(\eta_{1}, x\right)+c_{3}\left(\log \eta_{1}\right)^{\sigma-1}\right]-B_{1} I^{\alpha}\left(I^{\sigma} f(e, x)+c_{3}\right)}{\Delta_{2}}\right| \\
& \leq \frac{\left|c_{1}\right|+\left|A_{1}\right|\left|I^{\alpha+\gamma_{1}} \phi_{q}\left[\frac{\|p\|_{\infty} g(\|x\| \infty)\left(\log \eta_{1}\right)^{\sigma}}{\Gamma(\sigma+1)}+c_{3}\left(\log \eta_{1}\right)^{\sigma-1}\right]\right|+\left|B_{1}\right|\left|I^{\alpha}\left(\frac{\|p\|_{\infty} g\left(\|x\|_{\infty}\right)(\log (.))^{\sigma}}{\Gamma(\sigma+1)}+c_{3}\right)(e)\right|}{\left|\Delta_{2}\right|} \\
& \leq \frac{\left|c_{1}\right|+\left|A_{1}\right|\left|I^{\alpha+\gamma_{1}}\left[\frac{\|p\|_{\infty} g(\|x\| \infty)\left(\log \eta_{1}\right)^{\sigma}}{\Gamma(\sigma+1)}+c_{3}\left(\log \eta_{1}\right)^{\sigma-1}\right]^{q-1}\right|+\left|B_{1}\right|\left(\frac{\|p\|_{\infty} g\left(\|x\|_{\infty}\right) \Gamma(\sigma+1)}{\Gamma(\alpha+\sigma+1)}+\frac{c_{3}}{\Gamma(\alpha+1)}\right)}{\left|\Delta_{2}\right|} \\
& \leq \frac{\left|c_{1}\right|+\left|A_{1}\right|\left[\left|\left[\frac{\|p\|_{\infty} g\left(\|x\|_{\infty}\right)}{\Gamma(\sigma+1)}+c_{3}\right]^{q-1} I^{\alpha+\gamma_{1}}\left[\left(\log \eta_{1}\right)^{\sigma(q-1)}\right]+\right| B_{1} \mid\left(\frac{\|p\|_{\infty} g\left(\|x\|_{\infty}\right) \Gamma(\sigma+1)}{\Gamma(\alpha+\sigma+1)}+\frac{c_{3}}{\Gamma(\alpha+1)}\right)\right.}{\left|\Delta_{2}\right|} \\
& \leq \frac{\left|c_{1}\right|+\left|A_{1}\right|\left[\frac{\|p\|_{\infty} g\left(\|x\|_{\infty}\right)}{\Gamma(\sigma+1)}+c_{3}\right]^{q-1} \frac{\Gamma(\sigma(q-1)+1)\left(\log \eta_{1}\right)^{\alpha+\eta_{1}+\sigma(q-1)}}{\Gamma(\alpha+\gamma 1+\sigma(q-1)+1)}+\left|B_{1}\right|\left(\frac{\|p\|_{\infty} g\left(\|x\|_{\infty}\right) \Gamma(\sigma+1)}{\Gamma(\alpha+\sigma+1)}+\frac{c_{3}}{\Gamma(\alpha+1)}\right)}{\left|\Delta_{2}\right|} \\
& \leq \frac{\left|c_{1}\right|+\left|A_{1}\right|\left[\frac{\|p\|_{\infty} g\left(\|x\|_{\infty}\right)}{\Gamma(\sigma+1)}+c_{3}\right]^{q-1} \frac{\Gamma(\sigma(q-1)+1)}{\Gamma(\alpha+\gamma 1+\sigma(q-1)+1)}+\left|B_{1}\right|\left(\frac{\|p\|_{\infty} g\left(\|x\|_{\infty}\right) \Gamma(\sigma+1)}{\Gamma(\alpha+\sigma+1)}+\frac{c_{3}}{\Gamma(\alpha+1)}\right)}{\left|\Delta_{2}\right|} \\
& \leq \frac{\left|c_{1}\right|+\left(2^{q-1}-1\right)\left|A_{1}\right|\left[\frac{\|p\|_{\infty}^{q-1} g\left(\|x\|_{\infty}\right)^{q-1}}{\Gamma^{q-1}(\sigma+1)}+c_{3}^{q-1}\right] \frac{\Gamma(\sigma(q-1)+1)}{\Gamma(\alpha+\gamma 1+\sigma(q-1)+1)}+\left|B_{1}\right|\left(\frac{\|p\|_{\infty} g\left(\|x\|_{\infty}\right) \Gamma(\sigma+1)}{\Gamma(\alpha+\sigma+1)}+\frac{c_{3}}{\Gamma(\alpha+1)}\right)}{\left|\Delta_{2}\right|} \\
& \leq \frac{\left|c_{1}\right|+\left(2^{q-1}-1\right)\left|A_{1}\right|\left[\frac{\|p\|_{\infty}^{q-1} g\left(\|x\|_{\infty}\right)^{q-1}}{\Gamma^{q-1}(\sigma+1)}+c_{3}^{q-1}\right] \frac{\Gamma(\sigma(q-1)+1)}{\Gamma(\alpha+\sigma(q-1)+1)}+\left|B_{1}\right|\left(\frac{\|p\|_{\infty} g\left(\|x\|_{\infty}\right) \Gamma(\sigma+1)}{\Gamma(\alpha+\sigma+1)}+\frac{c_{3}}{\Gamma(\alpha+1)}\right)}{\left|\Delta_{2}\right|} .
\end{aligned}
$$

69 
It follows, from Inequalities (3.12), (3.13) and (3.14) that the inequality (3.11) becomes:

$$
\|Q(x)\| \leq \frac{\omega_{1}\left(2^{q-1}-1\right)}{\Gamma^{q-1}(\sigma+1)}\|p\|_{\infty}^{q-1} g\left(\|x\|_{\infty}\right)^{q-1}+\frac{\omega_{2}\|p\|_{\infty} g\left(\|x\|_{\infty}\right)}{\left|\Delta_{2}\right|}+\omega_{3},
$$

where:

$$
\begin{aligned}
& \omega_{1}=\frac{\Gamma(\sigma(q-1)+1)}{\Gamma(\alpha+\sigma(q-1)+1)}\left[1+\frac{\left|A_{1}\right|}{\left|\Delta_{2}\right|}\right]+\frac{\left(2^{q-1}-1\right)\left(\left|A_{2}\right|+\left|B_{2}\right|\right)^{q-1}}{\left|\Delta_{1}\right|^{q-1}} \\
& {\left[1+\frac{\left|A_{2}\right| \Gamma(\sigma(q-1)+1)}{\left|\Delta_{2}\right| \Gamma(\alpha+\sigma(q-1)+1)}\right]} \\
& \omega_{2}=\frac{\left|B_{1}\right| \Gamma(\sigma+1)}{\Gamma(\alpha+\sigma+1)}+\left(\frac{\left|A_{2}\right|+\left|B_{2}\right|}{\Gamma(\sigma+1) \Gamma(\alpha+1)\left|\Delta_{1}\right|}\right),
\end{aligned}
$$

and:

$$
\begin{aligned}
& \omega_{3}=\frac{\left|c_{1}\right|}{\left|\Delta_{2}\right|}+\frac{\left(2^{q-1}-1\right)^{2}\left|c_{2}\right|^{q-1}}{\left|\Delta_{1}\right|^{q-1}}\left(1+\frac{\left|A_{1}\right|}{\left|\Delta_{2}\right|}\right) \\
& +\frac{\left|c_{1}\right|\left|B_{1}\right|}{\left|\Delta_{1} \Delta_{2}\right| \Gamma(\alpha+1) \Gamma(\sigma+1)} .
\end{aligned}
$$

Consequently, as $x \in B_{r}$, we have:

$$
\|Q(x)\| \leq \mid \frac{\omega_{1}\left(2^{q-1}-1\right)}{\Gamma^{q-1}(\sigma+1)}\|p\|_{\infty}^{q-1} g(r)^{q-1}+\frac{\omega_{2}}{\Delta_{2}}\|p\|_{\infty} g(r)+\omega_{3} .
$$

Therefore, the Arzelá-Ascoli Theorem implies that $Q$ : $C([1, e], \mathbb{R}) \rightarrow C([1, e], \mathbb{R})$ is completely continuous. Let $x$ be a solution. Then, for $t \in[1, e]$, as in the first step, we have:

$$
\|x\|_{\infty} \leq \frac{\omega_{1}\left(2^{q-1}-1\right)}{\Gamma^{q-1}(\sigma+1)}\|p\|_{\infty}^{q-1} g\left(\|x\|_{\infty}\right)^{q-1}+\frac{\omega_{2}\|p\|_{\infty} g\left(\|x\|_{\infty}\right)}{\left|\Delta_{2}\right|}+\omega_{3},
$$

which implies that:

$$
\frac{\|x\|_{\infty}}{\frac{\omega_{1}\left(2^{q-1}-1\right)}{\Gamma^{q-1}(\sigma+1)}\|p\|_{\infty}^{q-1} g\left(\|x\|_{\infty}\right)^{q-1}+\frac{\omega_{2}\|p\|_{\infty} g\left(\|p\|_{\infty}\right)}{\left|\Delta_{2}\right|}+\omega_{3}} \leq 1 .
$$

In view of $\left(H_{4}\right)$, there exists $M_{2}$ such that $\|x\| 6=M_{2}$. Let us set:

$$
U=\left\{u \in C([1, e], \square): \mathrm{k} u \mathrm{k} \leq M_{2}\right\} .
$$

Note that the operator $Q: U \rightarrow C([1, e], \mathbb{R})$ is continuous and completely continuous. From the choice of $U$, there is no $u \in \partial \bar{U}$ such that $u=\lambda Q u$ for some $\lambda \in$ $(0,1)$. Consequently, by the nonlinear alternative of Leray- Schauder type (Lemma 2.5), we deduce that $Q$ has a fixed point $u \in U$ which is a solution of problem (1.1). This completes the proof.

\section{Examples}

Example 4.1 Consider the Problem

$$
\left\{\begin{array}{l}
D^{7 / 4}\left(\phi_{3 / 2}\left(D^{3 / 2} x(t)\right)\right)=f(t, x), \\
x(1)=\phi_{3 / 2}\left(D^{3 / 2} x(1)\right)=0, \\
I^{1 / 2} x(2)+2 x(e)=0, \\
I^{1 / 4}\left(\phi_{3 / 2}\left(D^{3 / 2} x\right)\right)(5 / 2)-\phi_{3 / 2}\left(D^{3 / 2} x\right)(e)=0,
\end{array}\right.
$$

where

$$
\sigma=\frac{7}{4}, \alpha=\frac{3}{2}, p=\frac{3}{2}, q=3, A_{1}=A_{2}=1, B_{1}
$$

$$
=2, B_{2}=-1, \gamma_{1}=\frac{1}{2}, \gamma_{2}=\frac{1}{4}, \eta_{1}=2, \eta_{2}=\frac{5}{2}, c_{1}=2
$$

and $c_{2}$

$=0$. Then, we have:

$$
\Delta_{1}=-0.157 \text { and } \Delta_{2}=2.61 \text {. }
$$

Consider problem (4.1) with:

$$
f(t, x)=\frac{1}{10} e^{-t^{2}} \sqrt{|x(t)|}+\arctan (1+t) .
$$

Clearly:

$$
|f(t, x)| \leq a(t)+b(t)\|x\|_{\infty},
$$

where, $a(t)=\arctan (1+t)$ and $b(t)=\frac{1}{10} e^{-t^{2}}$. It follows that:

$$
\|a\|_{\infty} \leq \frac{\pi}{2},\|b\|_{\infty}=\frac{1}{10 e} .
$$

With the given values, we find that:

$$
\theta_{1} \leq 8.58 \times 10^{-3} \text { and } \theta_{2} \leq 0.121 \text {. }
$$

So, $\theta_{1}+\theta_{2} \leq 1$. Thus, all hypothesis of Theorem 1.1 hold. Therefore, the Hadamard fractional integral boundary value problem (4.1) has at least one solution. 
Consider Problem (4.1) with:

$$
f(t, x)=\frac{\log t}{1+x^{2}} .
$$

It follows that:

$$
|f(t, x)| \leq p(t) g\left(\|x\|_{\infty}\right),
$$

for $p(t)=\log t$ and $g\left(\|x\|_{\infty}\right)=1$. Then, we have:

$$
\|p\|_{\infty}=1, \omega_{1}=488.4, \omega_{2}=6.405 \text { and } \omega_{3}=0.766
$$

Further, the hypothesis $\left(H_{4}\right)$, it is equivalent to show existence of $M_{2}$ such that:

$$
M_{2}>496 \text {. }
$$

Thus, all hypothesis of Theorem 1.2 hold. Therefore, the Hadamard fractional integral boundary value problem (4.1) has at least one solution.

\section{Example 4.2}

Consider the problem, for $\lambda>1$ :

$$
\left\{\begin{array}{l}
D^{\sigma}\left(\phi^{p}\left(D^{\alpha} x(t)\right)\right)=\frac{\lambda}{10} \log t x^{p-1}, \\
x(1)=\phi_{p}\left(D^{\alpha} x(1)\right)=0, \\
A_{1} I^{\gamma_{1}} x\left(\eta_{1}\right)+B_{1} x(e)=c_{1}, \\
A_{2} I^{\gamma_{2}}\left(\phi^{p}\left(D^{\alpha} x\right)\right)\left(\eta_{2}\right)+B_{2} \phi^{p}\left(D^{\alpha} x\right)(e)=c_{2}, .
\end{array}\right.
$$

1. Let, $\alpha=\sigma=7 / 4, p=5 / 4, q=5, A_{1}=B_{1}=-1 / 4, A_{2}=$ $B_{2}=1 / 4, c_{1}=1, c_{2}=0, \eta_{1}=2, \eta_{2}=5 / 2, \gamma_{1}=3 / 2$ and $\gamma_{2}$ $=5 / 4$. Then, it follows that:

$$
\begin{aligned}
& \|a\|_{\infty}=0,\|b\|_{\infty}=\frac{\lambda}{10}, \\
& \Delta_{1}=0.34, \Delta_{2}=-0.4, \\
& \theta_{1}=1.89\left(\frac{\lambda}{10}\right)^{4} \text { and } \theta^{2}=1.68 \frac{\lambda}{10} .
\end{aligned}
$$

The hypothesis $\left(\mathrm{H}_{2}\right)$ is equivalent to give a positive real $M_{1}$ such that:

$$
1.89\left(\frac{\lambda}{10}\right)^{4}+1.68 \frac{\lambda}{10} \leq 1
$$

So, for $\lambda<5$, there exist $M_{1}>0$ satisfying the above inequality. Thus, all hypothesis of Theorem 1.1 hold.
Therefore, the Hadamard fractional integral boundary value problem (4.1) has at least one solution:

2. Let $\alpha=3 / 2, \sigma=7 / 4, p=3, q=3 / 2, A_{1}=-1, B_{1}=2$, $A_{2}=1, B_{2}=0, c_{1}=c_{2}=0, \eta_{1}=5 / 2, \eta_{2}=3 / 2, \gamma_{1}=1 / 2$ and $\gamma_{2}=3 / 2$

It follows that:

$$
\begin{aligned}
|f(t, x)| & \leq p(t) g\left(\|x\|_{\infty}\right), \\
\text { for } p(t) & =\frac{\lambda}{10} \log \operatorname{tand} g\left(\|x\|_{\infty}\right)=\|x\|_{\infty}^{2} \text {. Then, we have } \\
\|p\|_{\infty} & =\mid \frac{\lambda}{10}, \omega_{1}=5.5, \omega_{2}=7 \text { and } \omega_{3}=0 .
\end{aligned}
$$

Further, the hypothesis $\left(H_{4}\right)$, it is equivalent to show existence of $M_{2}$ such that:

$$
0.7 \lambda M_{2}^{2}+(1.5 \sqrt{\lambda}-1) M_{2} \geq 0
$$

Then, for every $\lambda>1$, there exist $M_{2}>\frac{1.5 \sqrt{\lambda}-1}{0.7 \lambda}$. Thus, all hypothesis of Theorem 1.2 hold. Therefore, the Hadamard fractional integral boundary value problem (4.1) has at least one solution.

\section{Ethics}

This article is original and contains unpublished material. The corresponding author confirms that all of the other authors have read and approved the manuscript and no ethical issues involved.

\section{References}

Agarwal, R. P., \& O'Regan, D. (1998). Positive solutions to superlinear singular boundary value problems. Journal of Computational and Applied Mathematics, 88(1), 129-147. doi.org/10.1016/S0377-0427(97)00205-7

Granas, A., \& Dugundji, J. (2003). Elementary fixed point theorems. In Fixed Point Theory (pp. 9-84). Springer, New York, NY. doi.org/10.1007/978-0-387-21593-8

Baleanu, D., Diethelm, K., Scalas, E., \& Trujillo, J. J. (2012). Fractional calculus: Models and numerical methods (Vol. 3). World Scientific. doi.org/10.1142/8180

Chamekh, M., Ghanmi, A., \& Horrigue, S. (2018). Iterative approximation of positive solutions for fractional boundary value problem on the half-line. Filomat, 32(18), 6177-6187. doi.org/10.2298/FIL1818177C 
Ding, Y., Wei, Z., Xu, J., \& O’Regan, D. (2015). Extremal solutions for nonlinear fractional boundary value problems with p-Laplacian. Journal of Computational and Applied Mathematics, 288, 151-158. doi.org/10.1016/j.cam.2015.04.002

Ghanmi, A., \& Horrigue, S. (2018). Existence results for nonlinear boundary value problems. Filomat, 32(2), 609-618. doi.org/10.2298/FIL1802609G.

Ghanmi, A., \& Horrigue, S. (2019). Existence of positive solutions for a coupled system of nonlinear fractional differential equations. Ukrainian Mathematical Journal, 71(1), 39-49. doi.org/10.1007/s11253-019-01623-w

Guo, L. J., Sun, J. P., \& Zhao, Y. H. (2007). Multiple positive solutions for nonlinear third-order three-point boundary-value problems. Electronic Journal of Differential Equations (EJDE) [electronic only], 2007, Paper-No.

Guo, L. J., Sun, J. P., \& Zhao, Y. H. (2008). Existence of positive solutions for nonlinear third-order three-point boundary value problems. Nonlinear Analysis: Theory, Methods \& Applications, 68(10), 3151-3158. doi.org/10.1016/j.na.2007.03.008

Hadamard, J. (1892). Essay on the study of functions, given by their Taylor expansion. Gauthier-Villars.

Huang, H., \& Liu, W. (2018). Positive solutions for a class of nonlinear Hadamard fractional differential equations with a parameter. Advances in Difference Equations, 2018(1), 1-13. doi.org/10.1186/s13662-018-1551-9

Kilbas, A. A., Srivastava, H. M., \& Trujillo, J. J. (2006). Theory and applications of fractional differential equations (Vol. 204). elsevier.

Lakshmikantham, V., Leela, S., \& Devi, J. V. (2009). Theory of fractional dynamic systems. CSP.

Li, Y. L., \& Lin, S. Y. (2013). Positive Solution for the Nonlinear Hadamard Type Fractional Differential Equation with-Laplacian. Journal of Function Spaces and Applications, 2013. doi.org/10.1155/2013/951643

Liu, Z., Sun, J., \& Szántó, I. (2013). Monotone iterative technique for Riemann-Liouville fractional integro-differential equations with advanced arguments. Results in Mathematics, 63(3), 1277-1287. doi.org/10.1007/s00025-012-0268-4
Mâagli, H., Chaieb, M., Dhifli, A., \& Zermani, S. (2015). Existence and boundary behavior of positive solutions for a semilinear fractional differential equation. Mediterranean Journal of Mathematics, 12(4), 1265-1285. doi.org/10.1007/s00009-015-0571-X

Qi, T., Liu, Y., \& Zou, Y. (2017). Existence result for a class of coupled fractional differential systems with integral boundary value conditions. Journal of Nonlinear Sciences and Applications. JNSA, 10(7), 4034-4045. doi.org/10.22436/jnsa.010.07.52

Smart, D. R. (1974). Fixed point theorems, Cambridge Tracts in Mathematics, Cambridge University Press, London-NewYork, (1974).

Wang, G., \& Wang, T. (2016). On a nonlinear Hadamard type fractional differential equation with p-Laplacian operator and strip condition. Journal Nonlinear Science Application 9(7), 5073-5081. doi.org/10.22436/jnsa.009.07.10

Wang, G., Ghanmi, A., Horrigue, S., \& Madian, S. (2019). Existence result and uniqueness for some fractional problem. Mathematics, 7(6), 516. doi.org/10.3390/math7060516

Wang, G., Pei, K., Agarwal, R. P., Zhang, L., \& Ahmad, B. (2018). Nonlocal Hadamard fractional boundary value problem with Hadamard integral and discrete boundary conditions on a half-line. Journal of Computational and Applied Mathematics, 343, 230-239. https://doi.org/10.1016/j.cam.2018.04.062

Yang, X. J., Baleanu, D., \& Srivastava, H. M. (2015). Local fractional integral transforms and their applications. Academic Press. doi.org/10.1016/C2014-0-04768-5

Zhai, C., Wang, W., \& Li, H. (2018). A uniqueness method to a new Hadamard fractional differential system with four-point boundary conditions. Journal of Inequalities and Applications, 2018(1), 1-16. doi.org/10.1186/s13660-018-1801-0

Zhang, K., Wang, J., \& Ma, W. (2018). Solutions for integral boundary value problems of nonlinear Hadamard fractional differential equations. Journal of Function Spaces, 2018. doi.org/10.1155/2018/2193234 